\title{
A novel experimental method for the measurement of the caloric curves of clusters
}

\author{
Fabien Chirot $^{1,2}$, Pierre Feiden ${ }^{1,2}$, Sébastien Zamith ${ }^{1,2}$, Pierre Labastie ${ }^{1,2}$, and Jean-Marc L'Hermite ${ }^{1,2}$ \\ 1 Université de Toulouse ; UPS ; 118 route de Narbonne, F-31062 Toulouse, France \\ ${ }^{2}$ CNRS ; Laboratoire Collisions Agrégats Réactivité, IRSAMC ; F-31062 Toulouse, France
}

(Dated: November 6, 2018)

\begin{abstract}
A novel experimental scheme has been developed in order to measure the heat capacity of mass selected clusters. It is based on controlled sticking of atoms on clusters. This allows one to construct the caloric curve, thus determining the melting temperature and the latent heat of fusion in the case of first-order phase transitions. This method is model-free. It is transferable to many systems since the energy is brought to clusters through sticking collisions. As an example, it has been applied to $N a_{90}^{+}$and $N a_{140}^{+}$. Our results are in good agreement with previous measurements.

PACS numbers: 36.40.-c, 34.10.+x, 82.60.Nh
\end{abstract}

\section{INTRODUCTION}

Although melting is a universal property of bulk matter, no quantitative theory of this phenomenon is known. A possible way to tackle the problem is to study small systems, expecting that the number of degrees of freedom is low enough for the theory to be tractable. On the other hand, this makes comparison to experiments harder, since melting of very small particles cannot be seen directly. It has been shown however, that caloric curves could theoretically [1, 2, 3] and experimentally [4, 5] be used to characterize the melting transition. Generally, the melting temperatures of clusters are much lower than in the bulk, but higher temperatures have been reported for tin [6] and for gallium [7]. The most thorough study on small clusters is that of Schmidt et al [4]. They found that the melting temperature of small sodium particles (in the range of a hundred atoms) is far from monotonous as a function of size. The latent heat per atom also varies considerably. Those variations originate from a complex interplay between electronic, geometric and entropic effects [5, 8]].

The general scheme for cluster calorimetry is the following. Clusters are produced at a given temperature $T$ using a buffer gas as a heat bath. They are mass selected and then some energy $E$ is added to the clusters in order to bring them to a known reference state. An increase $\delta T$ of the temperature is compensated by a decrease $\delta E$ of the energy needed to reach the same state. The heat capacity is then deduced as the ratio $C(T)=\delta E / \delta T$. A peak in the curve $\mathrm{C}(\mathrm{T})$ is the signature of a phase transition. The maximum of this peak gives the melting temperature and its area is the latent heat [2].

In the existing experimental methods, the energy $E$ is brought either by a laser [4] or by collisions [7]. The method of Ref. 4 is accurate but requires a laser excitation. If photoabsorbtion is very efficient for metallic clusters, this is not the case for all kind of nanoparticles: most of them often undergo direct photodissociation rather than heating. This drawback can be overcome by the collisional technique [7]. There is price to pay however because the determination of the amount of energy transferred by inelastic collisions relies on a model.
Another method, which is not based on the determination of the caloric curve, has been used to extract melting temperatures [9]: one measures the mobility of clusters in a drift tube, from which collision cross sections are deduced. A variation of the cross section as a function of the temperature is the signature of the phase transition. This method is the only one able to detect a second-order phase transition. Nevertheless, it relies on a subtle effect that might be hard to detect. Since we can also measure collision cross sections in our experiment [10], we should also be able to detect the transition in a similar way, but preliminary measurements have not yet revealed any evidence of second order phase transition in sodium clusters.

This paper reports a new way to acquire caloric curves of mass selected clusters. Basically, as in previous experiments, we take advantage of the relation between evaporation rate and internal energy: the reference state, hence its internal energy, corresponds to a given evaporation rate. In our case the energy is brought by sticking collisions. In this way, unlike in Ref. 7, the energy is increased at each collision by a well defined quantity $E_{c}+D$, where $E_{c}$ is the collision energy and $D$ the dissociation energy. Moreover, as our method does not require laser excitation, it is easily transferable to many different systems. Furthermore, our method is parameter free: performing the experiment at two different collision energies allows us to get rid of the unknown dissociation energies in the final expression of the heat capacity. In this paper, we demonstrate the validity of our method using sodium clusters, in order to compare our results with those of Ref. 5 .

In the following, we first introduce the principle of the method used for extracting the caloric curves (Section (II), then we briefly describe the experimental setup (Section III). The evolution of the number of sticking in the multicollision regime is discussed in section IV] Section $\mathrm{V}$ is devoted to a detailed description of the method whose validity and robustness is discussed with the help of Monte Carlo simulations in section VI Finally, in section VII we show the experimental caloric curves obtained for $N a_{90}^{+}$and $N a_{140}^{+}$and compare them to previous results. 


\section{PRINCIPLE OF THE METHOD}

When performing calorimetry with nanoclusters, there is no way to measure the temperature after adding energy to the system, in contrast to macroscopic systems, where the temperature can be monitored as a function of added energy. Only the initial temperature can be fixed by a suitable preparation of the system. Another difficulty is related to the fact that measurements are performed on an assembly of clusters with some statistics involved. In order to illustrate the principle of the method, we neglect all statistical effects here. Further details are given in section $\mathrm{V}$

So we assume that all the clusters have initially a given internal energy $E_{0}(T)$. The purpose of the experiment is to add a known amount of energy $\Delta E$ to the cluster such as to bring it to a well defined and recognizable state. The energy $E_{f}=E_{0}(T)+\Delta E$ of this state is not necessarily known. All we need is that $E_{f}$ does not depend on the partition between $E_{0}$ and $\Delta E$. In our experiment, this final state is given by the condition that its lifetime is equal to the detection time in the experiment. Other experiments (see refs. 4, 7) use slight variations of this condition. The fact that this state is well defined is given by the theory of the evaporative ensemble 11]: since the lifetime with respect to evaporation depends exponentially on the energy, fixing the lifetime, even loosely, gives a well-defined value to the energy.

There are various ways to pour energy into the cluster. Haberland's group uses photons [4], Jarrold's uses inelastic collisions with a rare gas [7]. In our group, we use sticking collisions. In this case, the size $n$ is increased by one at each collision and the added energy is $D_{n+1}+E_{c}$, where $D_{n}$ is the dissociation energy of the cluster of size $n$ and $E_{c}$ the collision energy in the center of mass frame (it is easily shown that the rotational energy can be neglected). Basically, the energy $E_{f}$ is reached when no more sticking collision is possible, that is, the maximum number of sticking collisions depends on the initial energy. Fig. 1 illustrates this schematically. The solid line represents the internal energy $E_{0}(T)$ of the cluster as a function of its temperature. A first order phase transition shows up as a smooth jump in this curve (which gives a peak in the heat capacity). The vertical arrows in the figure represent the quantities $D_{n}+E_{c} . \quad E_{f}$ is depicted as constant, although it depends slightly on the size reached after the stickings have occurred. As the initial temperature is increased, the cluster can stand less and less sticking. Five situations are depicted where the number of sticking evolves from 5 to 1 . Before and after the phase transition, the number of sticking evolves slowly. But at the phase transition it evolves much more rapidly due to the rapid change in energy as the temperature increases.

Of course, the scheme presented on Fig. 1 is oversimplified. The main correction is that even if the initial energy is supposed to be well defined, the number of sticking collisions results from a statistical process, with

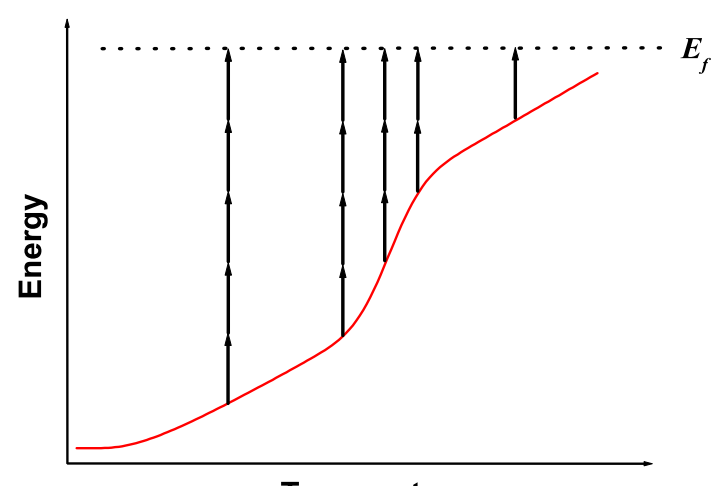

Temperature

FIG. 1: (Color on line) Illustration of the principle of our experiment. The cluster energy is plotted as a function of the initial temperature. Each small vertical arrow represents the quantity $D_{n}+E_{c}$. $E_{f}$ is the energy such that the dissociation time equals the observation time in our experiment. As the initial temperature is increased, less and less sticking are observed. This decrease in the number of sticking evolves more rapidly as a phase transition occurs.

Poisson probability. Furthermore, it is impossible to rule out evaporation during the process. For the sake of simplicity however, let us assume for the present discussion that we can extract from the experiment a well defined "maximum number of sticking" $n_{\max }$ for a given initial temperature. It is then theoretically possible to obtain the initial internal energy $E_{0}$ of a cluster of initial size $j$ as:

$$
E_{0}=E_{f}-\sum_{i=1}^{n_{\max }}\left(D_{j+i}+E_{c}\right)
$$

This equation cannot be used directly however, because neither $E_{f}$ nor the $D_{n}$ 's are known. We get rid of this problem by using a differential method. The basic idea is to measure the number of sticking $n_{\max }\left(T, E_{c_{1}}\right)$ and $n_{\max }\left(T, E_{c_{2}}\right)$ as a function of the initial temperature $T$ for two different collision energies $E_{c_{1}}$ and $E_{c_{2}}$, and to find two temperatures $T_{1}$ and $T_{2}$ such that $n_{\max }\left(T_{1}, E_{c_{1}}\right)=n_{\max }\left(T_{2}, E_{c_{2}}\right)$ (see Fig. 2). Then, $E_{f}$ and the $D_{n}$ 's are the same in both processes. This can be written as:

$$
\begin{aligned}
E_{f} & =E_{0}\left(T_{1}\right)+\sum_{i=1}^{n_{\max }}\left(D_{j+i}+E_{c_{1}}\right) \\
& =E_{0}\left(T_{2}\right)+\sum_{i=1}^{n_{\max }}\left(D_{j+i}+E_{c_{2}}\right)
\end{aligned}
$$

In a first approach, one can neglect the variation of the collisional energies with the number of sticking. By 


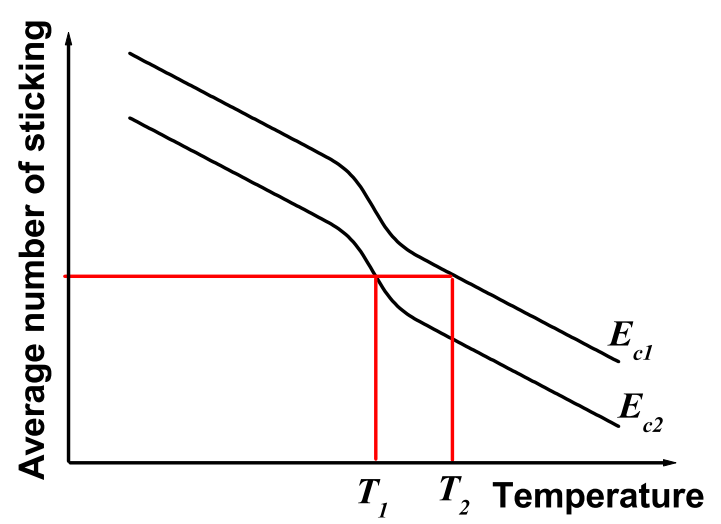

FIG. 2: (Color on line) Schematic view of the average number of sticking as a function of the temperature. Two curves are shown corresponding to two energies of collision $E_{c_{1}}$ and $E_{c_{2}}$, with $E_{c_{2}}>E_{c_{1}}$. When the collision energy is increased at a given temperature less sticking occurs.

subtracting Eq. 2 to Eq. 3 and using a finite differential approximation, one can derive:

$$
\frac{\partial E_{0}}{\partial T} \approx n_{\max } \frac{E_{c_{1}}-E_{c_{2}}}{T_{2}-T_{1}}
$$

Let us recall that this equation is a rough approximation however. It is useful for the understanding of the principle of the differential method, but it has been established with a number of simplifying hypotheses. Specially, the quantity $n_{\max }$ is not well defined. Instead, we will show in section IV that a well defined and relevant quantity is the average number of sticking. This quantity can be easily extracted from the experimental mass spectra after sticking.

\section{EXPERIMENTAL SETUP}

The experimental setup is depicted in Figure 3 and has already been described in details elsewhere [10]. The key points of the experiment are highlighted in the following.

The clusters are produced in a gas aggregation source. A crucible filled with sodium is heated up to $573 \mathrm{~K}$. The source walls are cooled down with liquid nitrogen. A controlled flux of cold Helium (173 K) flows through the hot sodium vapor and condensation occurs. A hollow cathode discharge in the crucible ionizes the produced clusters.

The helium buffer gas brings the sodium clusters to the next stage of the experiment, the thermalization chamber. Its temperature can be set between $140 \mathrm{~K}$ and $500 \mathrm{~K}$. Clusters thermalization occurs thanks to collision with the helium buffer gas $\left(\approx 10^{5}\right.$ collisions $)$. Out of the thermalization chamber flows a continuous beam of charged,

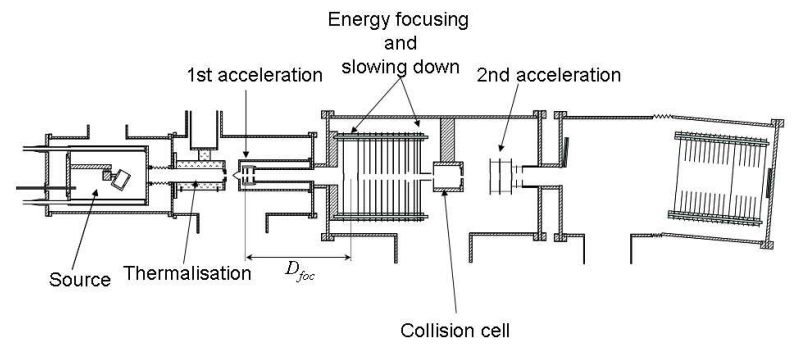

FIG. 3: Schematic view of the experiment.

temperature controlled, sodium clusters. The size distribution is controlled by varying the helium gas flow, the temperature of the crucible and the output diameter of the thermalization chamber. Typically, the center of the size distribution ranges from 30 to 300 atoms with a width of about 100.

We then apply pulsed voltages to electrodes in order to mass select, energy focus and slow down the clusters. The first acceleration stage consisting in 3 electrodes serves two purposes: first it allows a first raw mass selection, but more importantly, it is used to bring the clusters at the distance $D_{f o c}$ with a linear relation between their kinetic energy and their position. Clusters of different sizes will reach $D_{f o c}$ at different times. By applying a voltage on the energy focusing electrode at a specific time $T_{f o c}$, we compensate the energy dispersion for a given size of clusters, giving all of them the same energy to a very good accuracy.

Mass selected clusters are then decelerated with a gradual potential barrier until they reach the end of the slowing down apparatus. The voltages applied on electrodes are then suddenly shut down.

At this point, clusters are mass selected, thermalized and slowed down to a known controlled kinetic energy. They enter a cylindrical collision cell $(5 \mathrm{~cm}$ long, $2.5 \mathrm{~cm}$ radius, $5 \mathrm{~mm}$ entrance and exit holes). The vapor density $\rho$ of sodium atoms in the cell is controlled by the temperature $T_{c e l}$. After colliding, clusters finally reach the second acceleration stage, which, used in combination with a reflectron, gives us the size distribution at the output of the cell.

Clusters can be routinely slowed down to $E_{k}=10 \mathrm{eV}$. The full width at half maximum of the kinetic energy distribution is about $2 \mathrm{eV}$. The mean collision energy in the center of mass frame is given by

$$
E_{c}=\frac{E_{k}}{n+1}+\frac{3 n k_{B} T_{c e l}}{2(n+1)} .
$$

where the second term is the contribution from the mean kinetic energy of the atoms in the vapor. 
Our raw experimental data are the mass spectra recorded at the output of the cell, after parent clusters have undergone collisions. The mass spectra are recorded as a function of several parameters such as the initial temperature of the clusters, the density in the cell or the kinetic energy $E_{k}$.

The pulsed voltages are operated at $200 \mathrm{~Hz}$, and mass spectra are generally averaged over 6000 sweeps. The thermalizer or cell temperature is ramped $(1 \mathrm{~K} /$ minute) and mass spectra are continuously acquired with the following sequence:

- Mass spectrum at $E_{c_{1}}$

- Background spectrum at $E_{c_{1}}$

- Mass spectrum at $E_{c_{2}}$

- Background spectrum at $E_{c_{2}}$

until the final temperature is reached. Background peaks originate from masses close to the selected one, which, although not ideally transmitted through the energy focusing device, can also cross the collision cell and be detected. These peaks are present in the absence of sodium vapor in the cell. Background spectra are obtained by slightly changing $T_{f o c}$ so that neither the chosen mass nor any other mass is energy focused.

In the following section we analyze the evolution of the mass distributions at the output of the cell as the experimental parameters are varied.

\section{MASS DISTRIBUTIONS IN THE MULTICOLLISION REGIME}

When the thermalized, mass selected clusters fly through the cell, they undergo a number of collisions, depending on the density of atoms in the cell. If the collision energy is low enough, those collisions lead to the sticking of sodium atoms onto the clusters 12]. Fig. 4 shows the evolution of the size distribution at the exit of the cell when the atom density $\rho$ is increased. These spectra are obtained for $N a_{90}^{+}$slowed down to $E_{k}=20 \mathrm{eV}$ and thermalized at a temperature $T=146 \mathrm{~K}$. The top panel, for almost no density in the cell, demonstrates the ability of our setup to mass select the clusters $N a_{90}^{+}$. As can be seen, peaks appear after the selected mass. Particular care has been taken in order to eliminate these background peaks. Then, as the density is increased, bigger sizes appear. Since the collision energy is low $(0.25 \mathrm{eV}$ in the center of mass frame) compared to the dissociation energy (about $1 \mathrm{eV}$ ) and the clusters have a low temperature, several stickings are observed (up to 8 in the lower panel of Figure 4).

Starting from a cluster of size $j$, a convenient way to quantify the number of stickings, is to use the size distribution barycenter $\bar{n}$. It is calculated as:

$$
\bar{n}=\frac{\sum_{i} i \times I(i)}{\sum_{i} I(i)}
$$

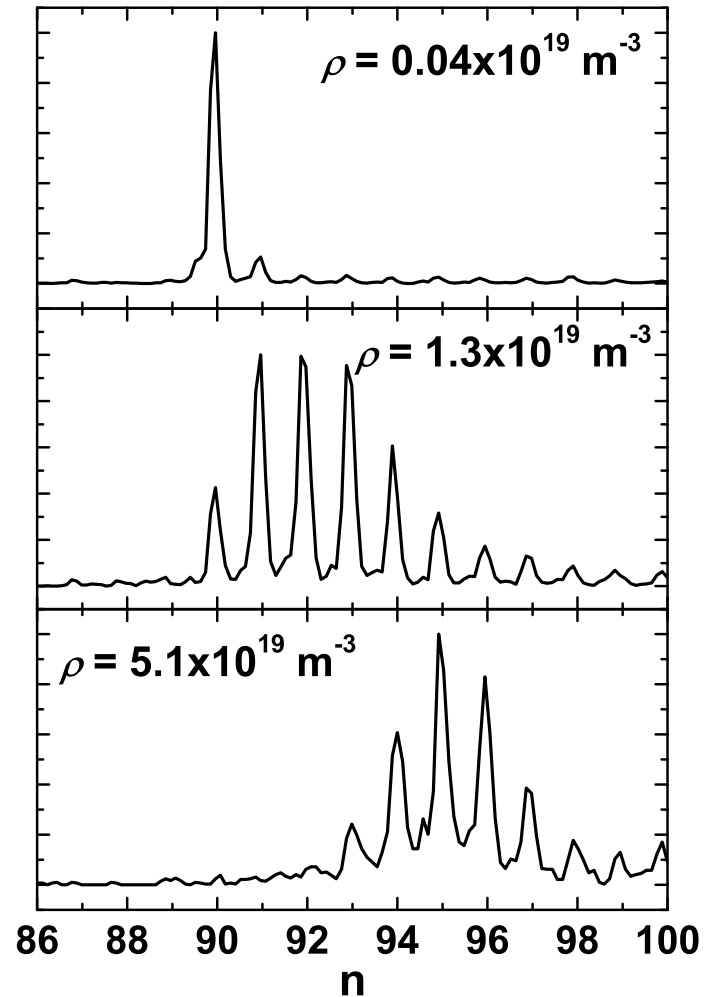

FIG. 4: Size distributions for $N a_{90}^{+}$clusters going through the cell with $T=146 \mathrm{~K}$ and $E_{k}=20 \mathrm{eV}$. As the density in the cell increases (from top to bottom), the number of sticking onto $N a_{90}^{+}$increases.

where $I(i)$ is the integrated signal of clusters of size $i+j$.

Fig. 5 shows the evolution of $\bar{n}$ as a function of the vapor density in the cell for $N a_{90}^{+}$with $E_{k}=20 \mathrm{eV}$ and $T=146 \mathrm{~K}$. The barycenter first increases until it reaches a plateau at $\bar{n} \approx 4.5$ for a density of $0.3 \times 10^{20} \mathrm{~m}^{-3}$. It then decreases continuously and can even reach negative values. Two competitive reactions occur in the cell that govern the evolution of the size distribution, the sticking of an atom onto the cluster:

$$
\begin{aligned}
& N a_{n}^{+}+N a \rightarrow N a_{n+1}^{+} \\
& E=E_{0}+D_{n+1}+E_{c}
\end{aligned}
$$

and the evaporation:

$$
\begin{array}{r}
N a_{n}^{+} \rightarrow N a_{n-1}^{+}+N a \\
E=E_{0}-D_{n}-\varepsilon
\end{array}
$$

where $E$ and $E_{0}$ are the internal energies after and before reaction respectively, $D_{n}$ are the dissociation energies of cluster of size $n, E_{c}$ is the collision energy in the center of mass frame and $\varepsilon$ the fragment kinetic energy. Three regimes can be distinguished:

1. The number of sticking increases linearly with the 


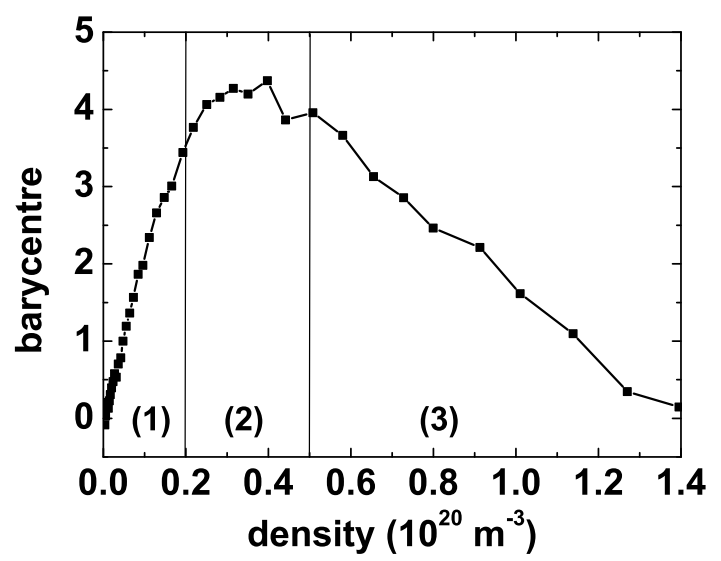

FIG. 5: Evolution of the sticking distribution barycenter as a function of the density of sodium atoms in the cell for $\mathrm{Na}_{90}^{+}$, $T=146 \mathrm{~K}$ and $E_{k}=20 \mathrm{eV}$. Three regimes can be distinguished: (1) linear increase, (2) saturation and (3) decrease.

density. Each collision leads to a sticking and $E$ remains low enough so that no evaporation occurs.

2. The number of sticking reaches a maximum (saturation). At each collision, the internal energy is increased by the dissociation energy $D_{n}$ plus the collision energy $E_{c}$. When the internal energy gets high enough, evaporation sets in and counterbalances the sticking: the mean evaporation time is nearly equal to the mean time between two collisions.

3. After saturation is reached, each extra collision leads to evaporation. Sticking-evaporation cycles increase the internal energy of the clusters by $E_{c}-\varepsilon$ $\left(\varepsilon \approx k_{B} T\right)$. Since $\varepsilon<E_{c}$, after a number of evaporation-sticking cycles, the clusters heat up and evaporation dominates.

The reliability of our method rests on the fact that the state of energy $E_{f}$ on Fig. 1 can be reached in the course of the flight through the cell. Thus, the density has to be high enough so that regime (2) or (3) occurs. This implies that evaporation occurs also during the process, so that the analysis leading to Eq. (44) has to be refined in order to account for evaporation. This is done in the next section.

\section{EXTRACTION OF THE CALORIC CURVES}

\section{A. Mathematical expression for the heat capacity}

Let us consider a cluster of initial energy $E_{0}$ and size $j$ when it enters the cell. Suppose now that it undergoes $i$ collisions during its flight through the cell, and that the final observed number of stickings is $n$, with $n<i$ because some evaporation occurs. Our basic hypothesis is that no matter the detailed history of these events, the final value of $n$ depends only on the total energy $E$ which has been brought to the cluster:

$$
E=E_{0}+i E_{c}+f
$$

where $f$ is an unknown quantity, which depends on the various values of the dissociation energies but neither on $E_{0}$ nor on $E_{c}$. There is no need in knowing $f$, as it does not appear in the final expression of the heat capacity. This hypothesis amounts to say that the number of evaporations is such as to bring back the cluster to the energy $E_{f}$ defined in section [I] and thus depends only on the excess energy $E-E_{f}$.

Now, all three quantities $E_{0}, E_{c}$ and $i$ are statistically distributed. In this treatment, we suppose that the widths of the $E_{0}$ and $E_{c}$ distributions are small respective to their averages, so that $E_{0}$ can be replaced by its average $E_{0}(T)$ and $E_{c}$ by its average Eq. (5). The main statistical effects come from the distribution of the number of collisions $i$, whose width is close to the average. The probability $P(i)$ is a Poisson law :

$$
P(i)=\frac{\bar{\imath}^{i} e^{-\bar{\imath}}}{i !}
$$

with an average $\bar{\imath}$, which can be easily computed knowing the density $\rho$ in the cell.

Computing the mean number of sticking, we obtain:

$$
\bar{n}=\sum_{i} P(i) n\left(E_{0}(T)+i E_{c}\right)
$$

By the implicit functions theorem, we have:

$$
\left.\frac{\partial E_{0}}{\partial E_{c}}\right|_{\bar{n}}=-\frac{\partial \bar{n}}{\partial E_{c}} / \frac{\partial \bar{n}}{\partial E_{0}}
$$

The two derivatives on the r.h.s. above are given by:

$$
\begin{aligned}
\frac{\partial \bar{n}}{\partial E_{0}} & =\sum_{i} P(i) \frac{\partial n}{\partial E}\left(E_{0}(T)+i E_{c}\right) \\
\frac{\partial \bar{n}}{\partial E_{c}} & =\sum_{i} i P(i) \frac{\partial n}{\partial E}\left(E_{0}(T)+i E_{c}\right)
\end{aligned}
$$

We now use a specific property of the Poisson law:

$$
i P(i)=\bar{\imath}\left(P(i)+\frac{\partial P(i)}{\partial \bar{\imath}}\right)
$$

so that Eq. (16) can be rewritten as:

$$
\frac{\partial \bar{n}}{\partial E_{c}}=\bar{\imath}\left(\frac{\partial \bar{n}}{\partial E_{0}}+\frac{\partial^{2} \bar{n}}{\partial E_{0} \partial \bar{\imath}}\right)
$$

So far, we neglected the change in cluster kinetic energy after each collision. When the number of collisions 


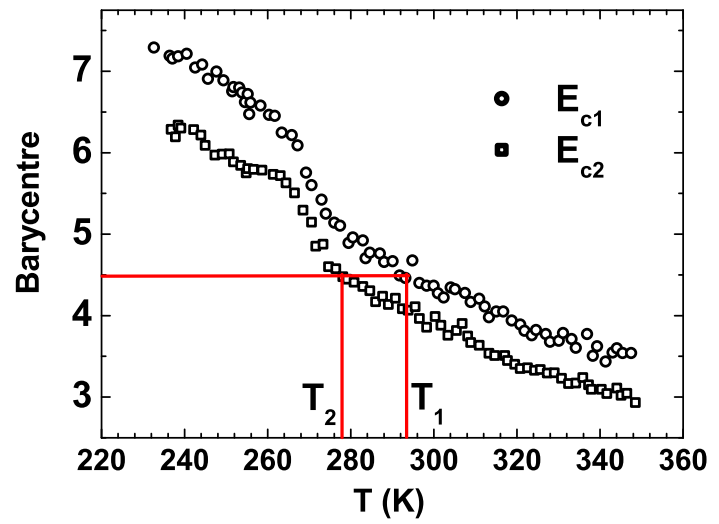

FIG. 6: (Color on line) Experimental data showing the barycenter $\bar{n}$ as a function of the initial temperature for $N a_{140}^{+}$ clusters. The two curves correspond to collision energies of $E_{c_{1}}=0.14 \mathrm{eV}$ and $E_{c_{2}}=0.21 \mathrm{eV}$

becomes large, this has to be accounted for through an effective value $E_{c}^{*}$, which is to a good approximation:

$$
E_{c}^{*}=E_{c} \frac{j+1}{\bar{\imath}}\left(1-\left(\frac{j}{j+1}\right)^{\bar{\imath}}\right)
$$

We use now

$$
C(T)=\frac{d E_{0}}{d T}
$$

together with equations (14) and (18), and we eventually obtain the final expression for the heat capacity:

$$
C(T)=-\left.\bar{\imath}\left(1+\frac{\partial^{2} \bar{n}}{\partial T \partial \bar{\imath}} / \frac{\partial \bar{n}}{\partial T}\right) \frac{\partial E_{c}^{*}}{\partial T}\right|_{\bar{n}}
$$

We describe in the next section how the different terms in Equation (21) are deduced from the experiment.

\section{B. Practical extraction of $C(T)$ from experimental curves}

In order to use Eq. (21), we need three partial derivatives and the value of $\bar{\imath}$. All these quantities can be extracted from experiment.

$$
\text { 1. The derivative }\left.\frac{\partial E_{c}}{\partial T}\right|_{\bar{n}}
$$

The method can be summarized as follows (see Fig. 6 for an illustration):

1. We record the barycenter $\bar{n}$ of the size distribution as a function of the initial cluster temperature for two different kinetic energies of the clusters $E_{c_{1}}$ and $E_{c_{2}}$.

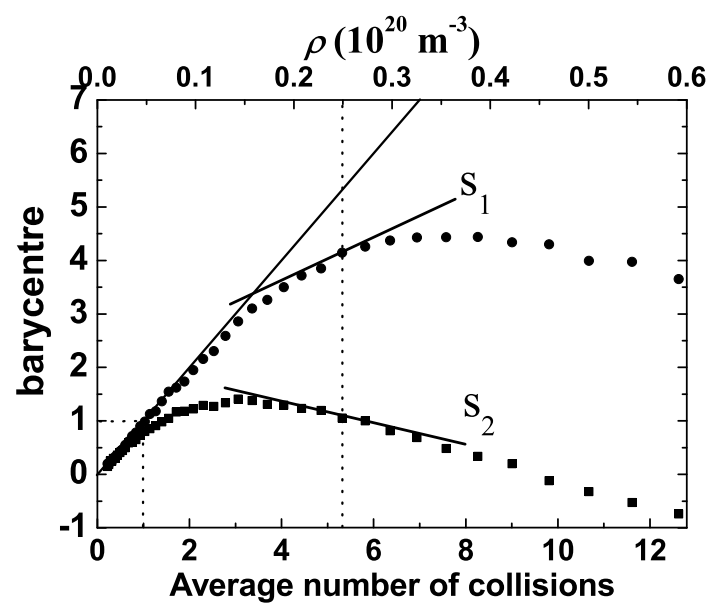

FIG. 7: Monte Carlo simulation of $\bar{n}(\rho)$ for two initial temperatures of $N a_{90}^{+}$clusters: $146 \mathrm{~K}$ (circles) and $293 \mathrm{~K}$ (squares) (see Section VI for details). The kinetic energy of the cluster is $20 \mathrm{eV}$. The bottom scale is in average number of collisions $\bar{\imath}$ whereas the top scale is in vapor density. At low density, the number of sticking is equal to the number of collisions. $S_{1}$ and $S_{2}$ are the slopes used in the determination of $\frac{\partial^{2} \bar{n}}{\partial \bar{\imath} \partial T}$.

2. We then chose a given value of the barycenter. For this value, we record the two corresponding temperatures $T_{1}$ and $T_{2}$ (as shown on Fig. 6).

3. We approximate:

$$
\left.\frac{\partial E_{c}}{\partial T}\right|_{\bar{n}}=\frac{E_{c_{2}}-E_{c_{1}}}{T_{2}-T_{1}}
$$

\section{Determination of $\bar{\imath}$}

The average number of collisions $\bar{\imath}$ can be considered to be the same for the two collision energies $E_{c_{1}}$ and $E_{c_{2}}$, as long as the difference $\delta E_{c}$ remains small enough (in other words, that the method is indeed differential). For instance, for clusters as small as $N a_{30}^{+}$at $E_{k}=15 \mathrm{eV}$ and $20 \mathrm{eV}$ (that correspond to 0.53 and $0.70 \mathrm{eV}$ collision energies) and for a density $\rho=0.2 \times 10^{20} \mathrm{~m}^{-3}$ in the cell, Monte Carlo simulations (see next section) show that the average number of collisions are respectively 2.11 and 2.08. As one can see, the difference is small enough that it can be neglected. Note that as the cluster size increases it becomes even easier to fulfill this requirement.

The average number of collisions $\bar{\imath}$ is deduced from saturation curves such as the ones presented in Figure 7. There is no need in knowing the value of the cross sections to determine this parameter. The main reason is that in regime (1), each collision leads to a sticking. The proportionality factor between the cell density $\rho$ and $\tau$ is thus deduced from the initial slope of the curves. Actually collisions at high impact parameter might not lead 


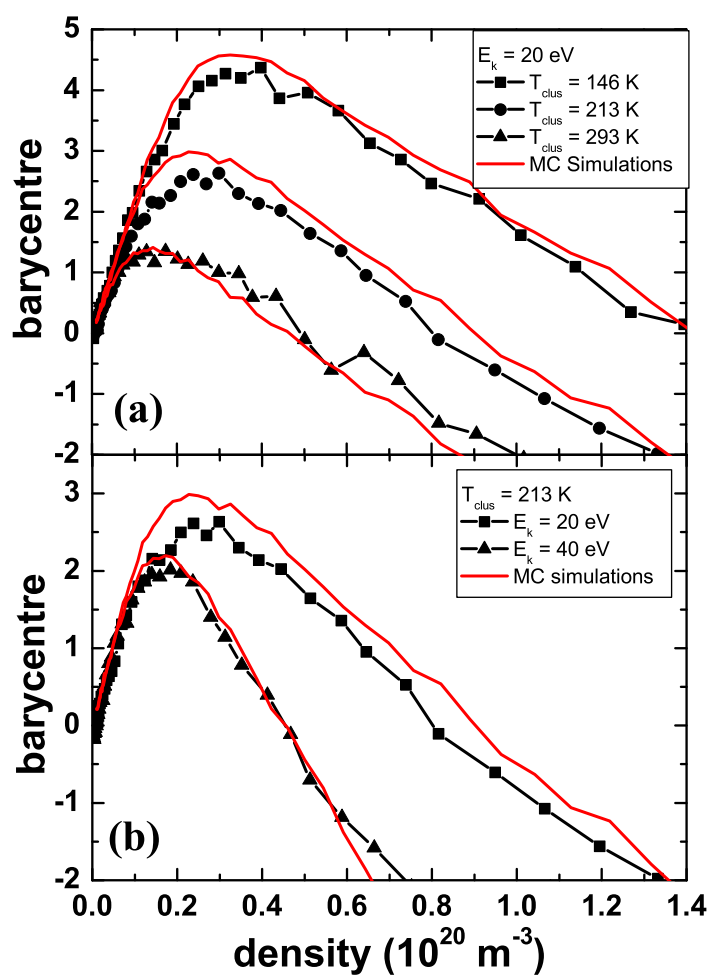

FIG. 8: (Color on line) Evolution of the size distribution barycenter as a function of the density of sodium atoms in the cell for $N a_{90}^{+}$for (a) three different initial temperatures (the kinetic energy is $E_{k}=20 \mathrm{eV}$ ) and (b) two different kinetic energies $(T=213 \mathrm{~K})$. Full lines are the results of Monte Carlo simulations (see Section VI).

to sticking. These collisions involve a very small energy exchange anyway, so that they can be neglected[12].

3. The derivatives $\frac{\partial^{2} \bar{n}}{\partial T \partial \bar{\imath}}$ and $\frac{\partial \bar{n}}{\partial T}$

The principle is presented on Fig. 7 too. The slopes $S_{1}$ and $S_{2}$ are the derivatives $\frac{\partial \bar{n}}{\partial \bar{\imath}}$ at temperatures $T_{1}$ and $T_{2}$ respectively. $\frac{\partial^{2} \bar{n}}{\partial T \partial \bar{\imath}}$ is then estimated as $\left(S_{2}-S_{1}\right) /\left(T_{2}-\right.$ $\left.T_{1}\right)$. Note that its value does not depend on the collision energy (which needs nevertheless to be the same for the two curves at $T_{1}$ and $T_{2}$ ).

Finally the derivative $\frac{\partial \bar{n}}{\partial T}$ is evaluated using the curves $\bar{n}(T)$ (see Fig. 6).

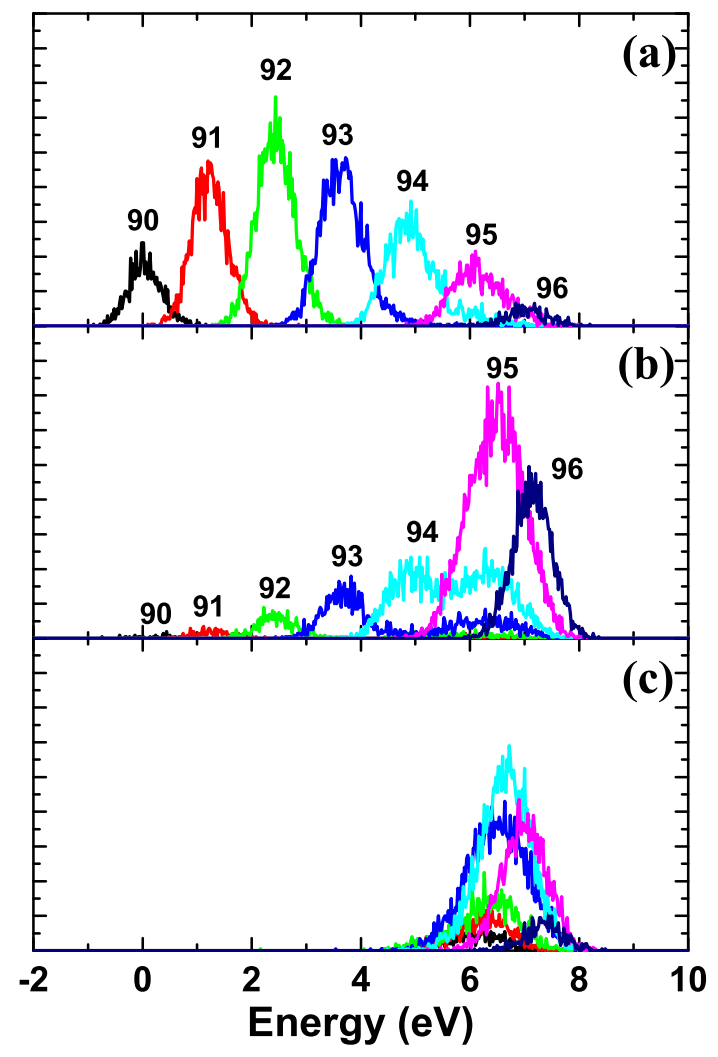

FIG. 9: (Color online) Simulation of the internal energy distribution for three different densities in the cell: (a) 0.1, (b) 0.32 and (c) $0.72 \times 10^{20} \mathrm{~m}^{-3}$. The initial temperature of the clusters is $146 \mathrm{~K}$ and $E_{k}=20 \mathrm{eV}$. Peaks are labeled by the size of the clusters.

\section{MONTE CARLO SIMULATION: VALIDATION OF THE METHOD}

\section{A. Description of the simulations}

Realistic Monte Carlo simulations have been performed in order to check the validity of the method under real conditions where the above analytical ideal approach might be invalidated by a number of details that are not taken into account: initial internal and collisional energy distributions, spatial divergence due to collisions, variations of $\frac{\partial^{2} \bar{n}}{\partial T \partial \bar{\imath}}$, dissociation events between the output of the cell and the detector,...

The initial internal energy of the clusters is randomly picked such that the average energy is equal to:

$$
\langle E\rangle=\frac{(3 j-6) h \nu_{0}}{e^{\frac{h \nu_{0}}{k_{B}{ }^{T}}}-1}
$$

where $j$ is the number of atoms in the cluster, $h$ the Planck constant, $\nu_{0}$ the vibration frequency. The frequency $\nu_{0}$ is taken from 13 and is equal to $2.3 \times 10^{12} \mathrm{~Hz}$. 
The phase transition is taken into account by adding the latent heat $L_{j}(T)$ to $\langle E\rangle$ in the following way assuming a gaussian peak in the heat capacity:

$$
L_{j}(T)=L_{j 0} \frac{\left(1-\operatorname{erf}\left(-\frac{\left(T-T_{j f}\right)}{\Delta T_{j}}\right)\right)}{2}
$$

where $T_{j f}$ is the temperature of the phase transition, $\Delta T_{j}$ is the width of the phase transition and erf is the error function. $L_{j 0}$ is the latent heat for a cluster of size $j$. This way we simulate the broadening of the phase transition for a finite system.

The evaporation rate is estimated using a Weisskopf approximation:

$$
\Gamma_{\text {evap }}=8 \pi \nu_{0}(3 j-7) \sigma \frac{\left(1-D_{j} / E\right)^{3 j-8}}{E}
$$

where $\sigma$ is the sticking cross section to the cluster of size $j-1$. The collision rate is given by:

$$
\Gamma_{\text {coll }}=\rho \sigma v_{\text {rel }} .
$$

where $v_{\text {rel }}$ is the relative velocity of the cluster and the sticking atom. The velocity distribution of atoms is assumed to follow a Boltzman distribution.

At each collision, the change in speed of the clusters is taken into account. Since only clusters with the right velocity are detected, clusters that undergo a lot of collisions become too slow to be detected and are discarded in the simulation.

We first checked that our Monte Carlo simulations could reproduce the experimental results of Figs. 5 and 8 , The agreement between the simulations and the experimental data is good. In order to reproduce correctly the experimental results it is important to put in the correct latent heat and melting temperature for $N a_{90}^{+}$. We took $T_{f}=205 \mathrm{~K}$ and $L=8 \mathrm{meV} /$ atom for the melting temperature and the latent heat respectively. On the other hand, for the other sizes the latent heat and temperature of fusion can be chosen arbitrarily: there is no change in the curve $\bar{n}(\rho)$.

For the dissociation energies, only values of $D_{n}$ up to $\mathrm{n}=37$ [14] are available. In the simulations presented here, the dissociation energies are all taken equal to $0.94 \mathrm{eV}$.

These simulations allow us to follow the evolution of the internal energy distribution of the clusters as a function of their size as the density in the cell is varied. In Figure 9 we present the energy distribution of the clusters for three different densities in the cell. In Figure 9(a) the density in the cell is $0.1 \times 10^{20} \mathrm{~m}^{-3}$, corresponding to an average number of sticking $\bar{n} \approx 3$ (in this case evaporation is completely negligible so that each collision leads to a sticking). We see in the figures well separated peaks corresponding to the different masses obtained after the cell. The energy separation between the peaks correspond to the dissociation energy plus the collision energy. The width of the peaks originates mainly from the initial canonical energy distribution. This can be seen from the $n=90$ energy distribution: indeed, in this example part of the parent clusters did not collide nor evaporate. Nevertheless, as the cluster size increases we observe an increase in the width. This comes from the width of the collision energy distribution which adds at each new collision.

In Figure 9(b), the density is $0.32 \times 10^{20} \mathrm{~m}^{-3}(\bar{\imath} \approx 7)$. This corresponds to the saturated case (regime $(2)$ of Figure 5). If we consider the sizes $n=93$ and 94, the internal energy distribution becomes bimodal: the high energy part comes from the evaporation of bigger clusters. For the two biggest sizes, namely $n=95$ and 96 , one can see as well that the energy separation between the peaks gets smaller. In fact, there is a strong contribution coming from the evaporation of $n=96$ clusters in the internal energy distribution of the $n=95$ clusters. For the size $n=96$, there is of course also contribution from the evaporation of bigger sizes that are not observed. Indeed due to their high evaporation rate, they evaporate before detection.

As the density is increased to $0.72 \times 10^{20} \mathrm{~m}^{-3}$ (Figure 9(c)), all clusters end up with almost the same internal energy. In this last case the average number of collisions is about 17 , much more than the maximum number of sticking observed (which is about 6 ). The observed internal energy is the maximum energy clusters can bear without evaporating before detection. In this case the observed clusters have all undergone evaporation.

Despite the complicated evolution of the internal energy of the clusters as the number of collision increases, the size distribution, for a given collision energy and density in the cell, is still essentially governed by the internal energy of the incoming cluster. Furthermore, as shown below, the fact that clusters of a specific size don't have necessarily a well defined internal energy do not prevent the determination of the caloric curves.

\section{B. Test of the method of section $\mathrm{V}$}

In order to test the method of section $\mathrm{V}$, we start from a theoretical caloric curve for $N a_{90}^{+}$and we perform a set of simulations that mimics the experimental curves. Then, using these fake experimental curves, we apply the method and we extract a caloric curve, which is then compared to the theoretical one we started with.

First of all, two saturation curves at $E_{k}=20 \mathrm{eV}$ were simulated for two initial temperatures of the clusters, namely $146 \mathrm{~K}$ and $293 \mathrm{~K}$. These curves are presented in Fig 7 and, as already shown, allow to calculate the various terms involved in Eq. (21).

Mass spectra were then simulated at $E_{c} \approx 0.4$ and $0.5 \mathrm{eV}$ as a function of the initial temperature of $N a_{90}^{+}$ clusters. 5000 trajectories were simulated at each temperature. Two set of simulations were performed at two different vapor densities, corresponding respectively to $\bar{\imath}=5.3$ and $\bar{\imath}=10.6$. In the first case the working density 


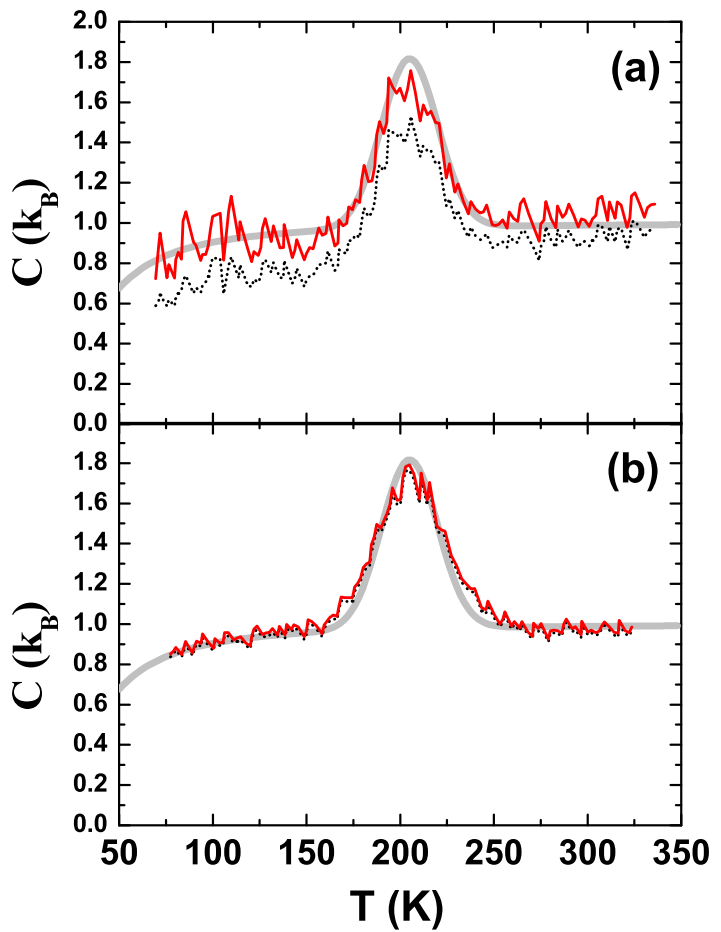

FIG. 10: (Color online) The thick solid line is the caloric curve introduced in the simulation $\left(L=8 \mathrm{meV} /\right.$ atom, $T_{f}=205 \mathrm{~K}$ and $\Delta T=30 \mathrm{~K})$. The dotted curves correspond to the caloric curves obtained by setting the second derivative in Eq. 21 to 0 whereas for the curves in thin solid line it is taken into account. The top panel correspond to $\bar{\imath}=5.3$ and the bottom one to $\bar{\imath}=10.6$

roughly corresponds to the maximum of the saturation curve (see Fig 7). It would not be a very good choice in a real experiment since the curvature of $\frac{\partial n}{\partial \bar{l}}$, thus the value of the second derivative in Eq. (21), is maximum. For $\bar{\imath}=10.6$, the second derivative in Eq. (21) is much smaller because the saturation curves already become much more parallel (see Fig 7).

Figure 10 shows a comparison between the theoretical caloric curve introduced in the calculation and the curves deduced from the simulation of the experiment. Fig. 10(a) corresponds to $\bar{\imath}=5.3$ and Fig. 10(b) to $\bar{\imath}=10.6$.

In order to evaluate the effect of the second derivative in Eq. (21), two curves are represented for each vapor density in each panel of Fig. 10, respectively with and without this term. For $\bar{\imath}=10.6$, within the noise, the curves are virtually indistinguishable. On the other hand, for $\bar{\imath}=5.3$, although the general shape of the caloric curve is correctly obtained with or without the second derivative, a quantitative agreement is obtained only by having it taken into account. The correction nicely improves the curve even in this unfavorable case. Nevertheless the smallest the correction the more accu- rate the result. Note that the noise is more important for $\bar{\imath}=5.3$ than for $\bar{\imath}=10.6$ : since the mean energy difference is roughly $\bar{\imath} \delta E_{c}$, the two curves $\bar{n}(T)$ get closer for small $\bar{\imath}$ values.

As already mentioned the simulations presented here are done with all clusters having the same dissociation energies. Nevertheless, we carefully checked that the introduction of magic numbers (i.e. higher dissociation energies) for arbitrary sizes does not influence our determination of the caloric curve.

Furthermore we performed simulations with randomly chosen temperature of fusion, latent heat and width of the transition for cluster sizes other than the initial one, and again this does not affect our determination of the latent heat and temperature of fusion of the initial cluster size.

From these simulations, we find our method particularly robust. The simulations demonstrate that the average number of sticking $\bar{n}$ is a well suited quantity to characterize the size distribution, even when evaporation dominates, provided Eq. (21) is used.

\section{EXAMPLES OF EXPERIMENTAL RESULTS WITH $N a_{90}^{+}$AND $N a_{140}^{+}$}

We present in Figure 11 the experimental caloric curves obtained for $\mathrm{Na}_{90}^{+}$and $\mathrm{Na}_{140}^{+}$clusters. The melting temperature is obtained from these curves as the maximum of the peak. The latent heat is deduced by integrating the peak area, after removing the base line (grey filled area under the curves in figure 11). The caloric curve for $N a_{90}^{+}$was not measured previously. We find a melting temperature of $205 \pm 5 \mathrm{~K}$ and a latent heat per atom of $8 \pm 2 \mathrm{meV}$.

For the $N a_{140}^{+}$clusters, the melting temperature is $272 \pm 4 \mathrm{~K}$ and the latent heat is $14 \pm 2 \mathrm{meV} /$ atom. This is consistent with previous measurements by Schmidt et al [5] $\left(T_{\text {fus }}=262 \mathrm{~K}, L=11 \mathrm{meV} /\right.$ atom $)$.

The results for $N a_{90}^{+}$are quite noisy: this is due to the relatively small latent heat of this cluster and the fact that the width of the transition is relatively large. Nevertheless we can still identify a peak in the caloric curve for this disfavoring case. We observe that the latent heat increases at high temperatures. Such an increase has already been observed, although less pronounced (see 44] for $N a_{192}^{+}$and [15] for $N a_{139}^{+}$). It might be due to either a rising background due to a high Debye temperature or a melting occurring in several steps. However the noise level is such that we can not confirm the significance of this increase in our case.

As shown from Fig. 10 the exact choice of the vapor density in the cell is not critical. Nevertheless, an optimal choice exists. As already mentioned in Sec. VI, it can be chosen so that it minimizes the noise. In order to minimize the second derivative in Eq. (21), the vapor density $\rho$ has to be chosen high enough such that the curves $\bar{n}(\rho)$ become almost parallel. On the other hand, if $\rho$ be- 


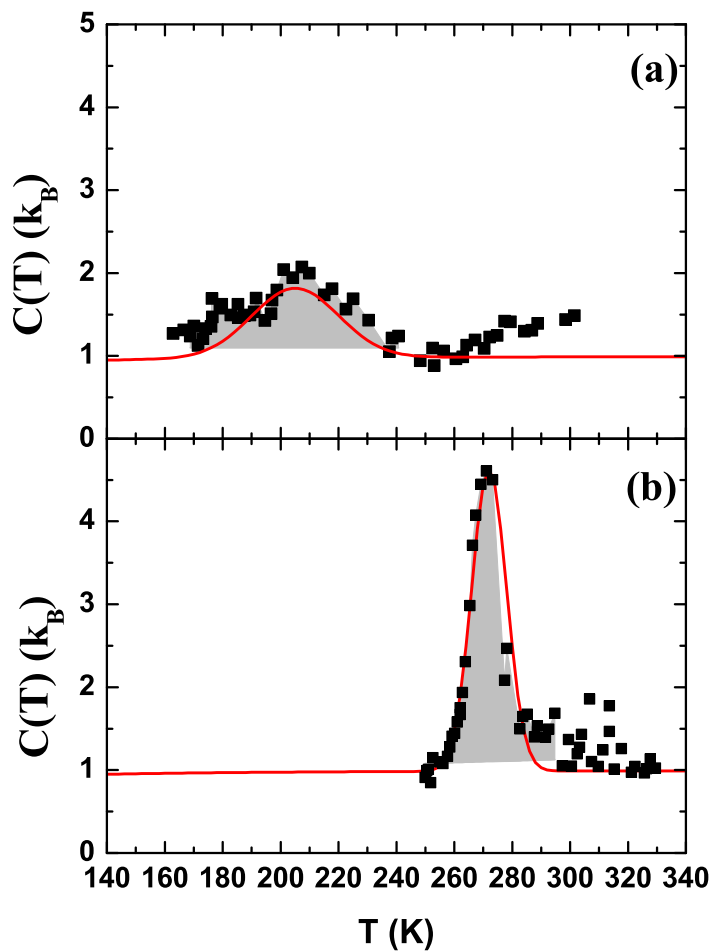

FIG. 11: (Color online) Experimental caloric curves for (a) $N a_{90}^{+}$and (b) $N a_{140}^{+}$clusters. $C(T)$ is in unit of $k_{B}$ per degree of freedom. The full lines represent the latent heat used in the simulations (see Eq. 24) and are used to guide the eye. The grey filled area under the curves represent the latent heat.

comes too large, evaporation becomes too strong and the signal gets scattered over many mass peaks, which is unfavorable for the statistics. Furthermore the background pressure in the collision chamber gets too high and leads to unwanted collisions out of the cell. Finally, too large $\bar{\imath}$ result in angular scattering of the clusters.

The use of a differential method has several advantages. First, we do not need to know the dissociation energies. Second, the presence of magic numbers is not a problem with this method. Indeed, any accident in the curves $\bar{n}(T)$ is canceled out by the differentiation. These all in one make this method reliable and precise. On the other hand, noise is a drawback. Since we rely on the calculation of finite differences for the determination of the caloric curves, and that the experimental data is discrete, the numerical differentiation is inherently noisy.

\section{CONCLUSION}

We have developed a novel experimental method for the measurement of caloric curves of small nanoparticles based on the sticking of atoms onto clusters. We have mathematically established the method and shown with the help of simulations that it is extremely robust. It associates the accuracy of the photoexcitation methods and the large application field of collisional methods. Moreover, its principle makes it rigorously independent on preliminary assumptions about properties such as dissociation energies or collisional energy transfer.

We have experimentally demonstrated the use of the method on sodium clusters (namely $N a_{90}^{+}$and $N a_{140}^{+}$).

\section{Acknowledgments}

This work has been partly funded by the Agence Nationale de la Recherche under grant ANR-05-BLAN0145. We gratefully acknowledge M. Schmidt for fruitful discussions.
[1] H.-L. Davis, J. Jellinek, and R.-S. Berry, J. Chem. Phys. 86, 6456 (1987).

[2] M. Bixon and J. Jortner, J. Chem. Phys. 91, 1631 (1989).

[3] P. Labastie and R. L. Whetten, Phys. Rev. Lett. 65, 1567 (1990).

[4] M. Schmidt, R. Kusche, B. von Issendorf, and H. Haberland, Nature (London) 393, 238 (1998).

[5] H. Haberland, T. Hippler, J. Donges, O. Kostko, M. Schmidt, and B. von Issendorf, Phys. Rev. Lett. 94, 035701 (2005).

[6] A. A. Shvartsburg and M. F. Jarrold, Phys. Rev. Lett. 85, 2530 (2000).

[7] G. A. Breaux, R. C. Benirschke, T. Sugai, B. S. Kinnear, and M. F. Jarrold, Phys. Rev. Lett. 91, 215508 (2003).

[8] A. Aguado and J. M. Lopez, Phys. Rev. Lett. 94, 233401 (2005).
[9] G. Breaux, B. Cao, and M. Jarrold, J. Phys. Chem. B 109, 16575 (2005).

[10] F. Chirot, S. Zamith, P. Labastie, and J.-M. L'Hermite, Rev. Sci. Instrum. 77, 063108 (2006).

[11] C. Klots, Z. Phys. D 5, 83 (1987).

[12] F. Chirot, S. Zamith, P. Labastie, and J.-M. L'Hermite, Phys. Rev. Lett. 99, 193401 (2007).

[13] W. A. de Heer, W. D. Knight, M. Y. Chou, and M. L. Cohen, in Solid State Physics, edited by F. Seitz and D. Turnbull (Academic, New York, 1987), vol. 40, p. 93.

[14] C. Bréchignac, P. Cahuzac, J. Leygnier, and J. Weiner, J. Chem. Phys. 90, 1492 (1989).

[15] R. Kusche, T. Hippler, M. Schmidt, B. von Issendorff, and H. Haberland, Eur. Phys. J. D 9, 1 (1999). 